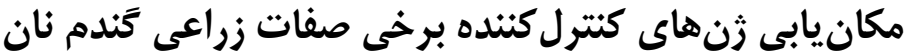

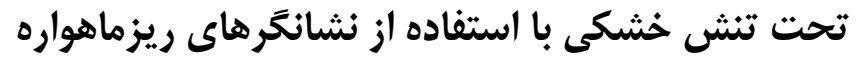

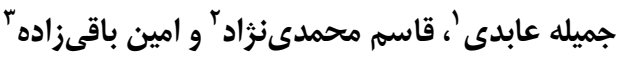

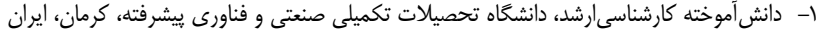

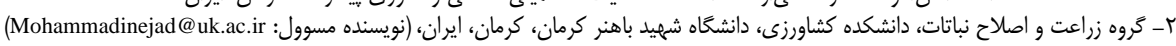

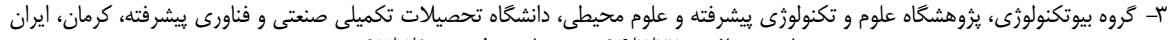
تاريخ دريافت:

صفحه: •19 19V تان

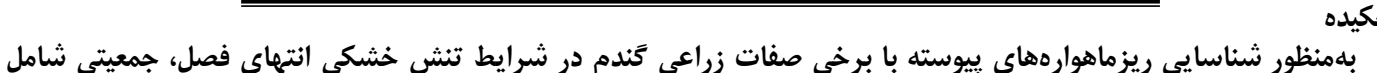
7 9 خانواده F:

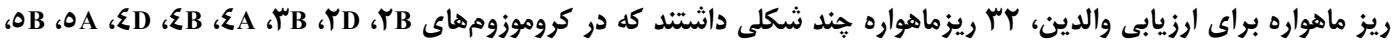
مكان يابى ، CD

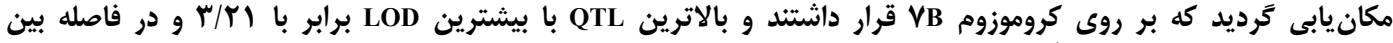

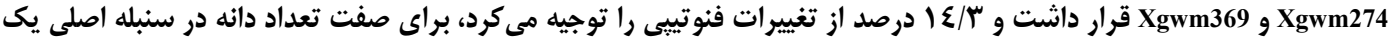

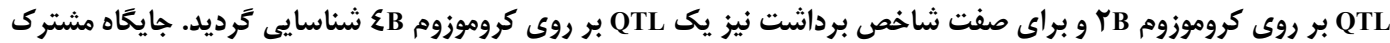

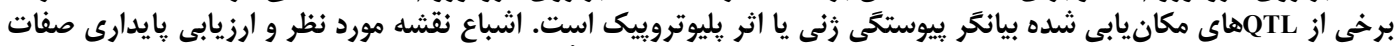

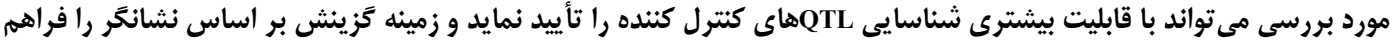

نمايد.

وازمهاى كليدى: تنش خشكى، كندم نان، QTL،SSR

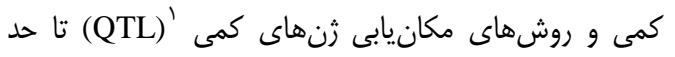

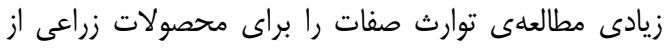

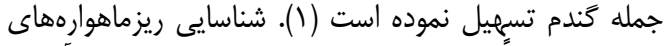

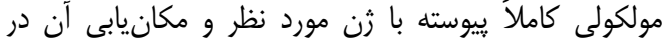

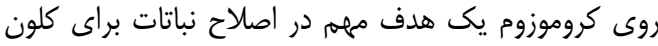

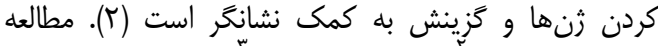

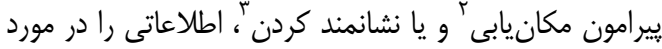

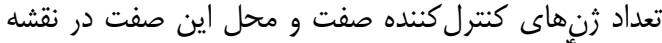

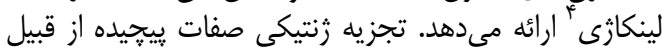

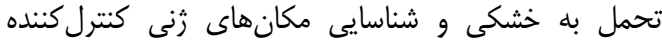

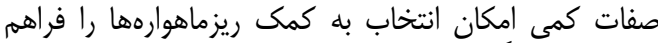

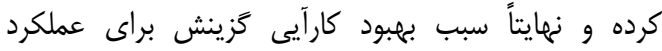

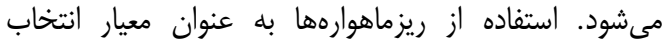

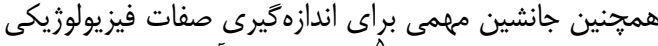

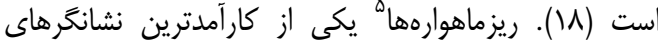

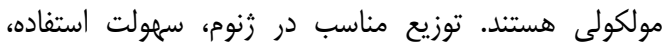

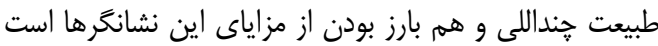

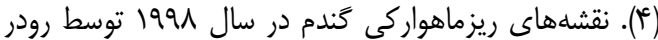

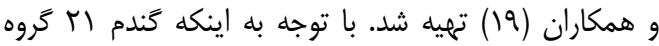

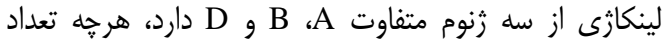

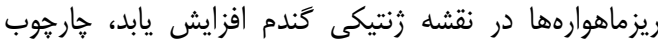

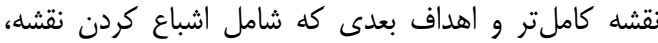

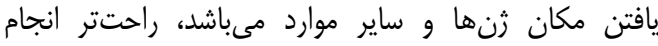

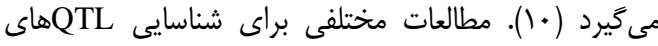

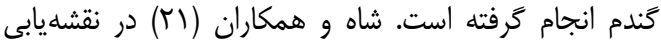

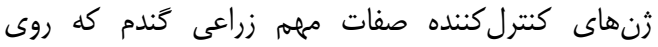

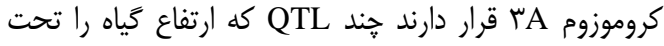

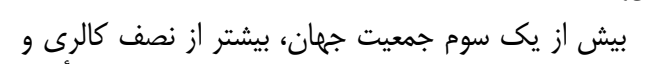

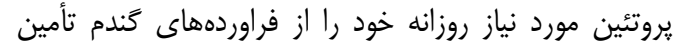

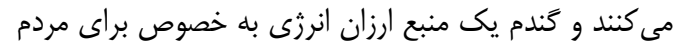

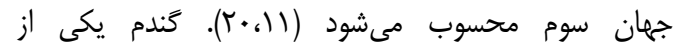

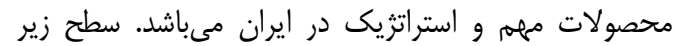

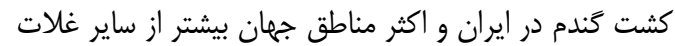

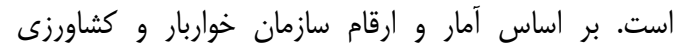

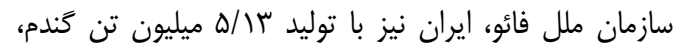

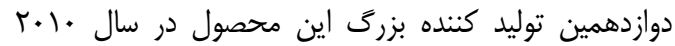

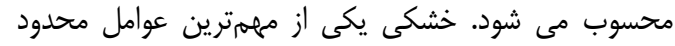

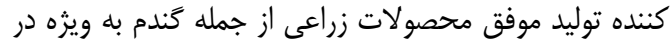

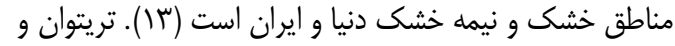

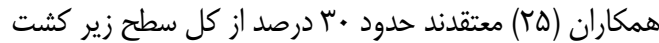

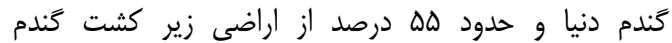

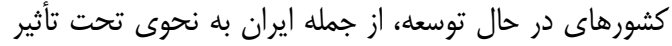

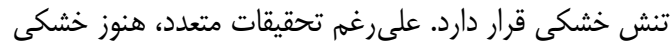

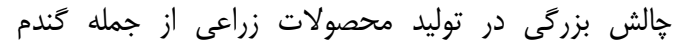

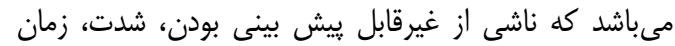

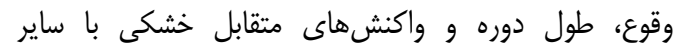

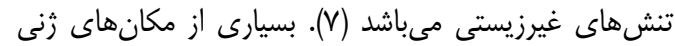

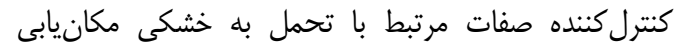

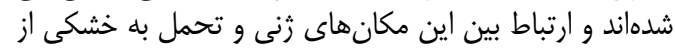

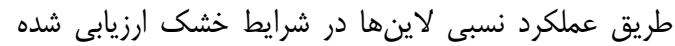

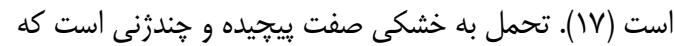

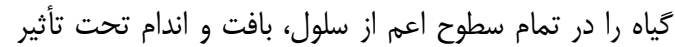

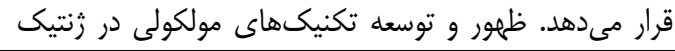

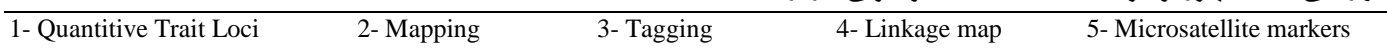


سانتى

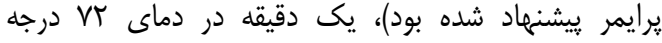
سانتى

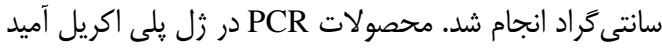

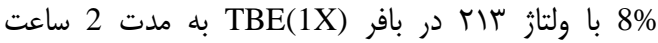

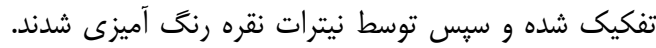

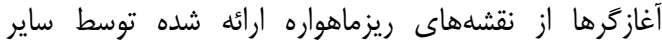

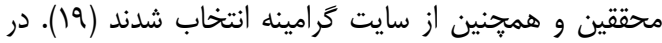

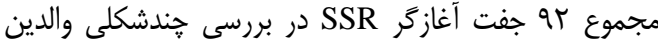

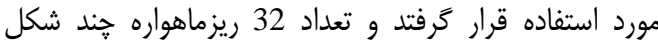

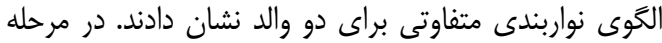

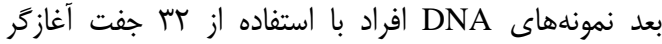

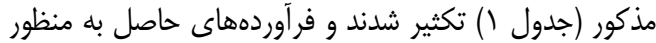
تعيين زنوتيڤ افراد، الكتروفورز شدند

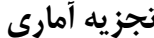

براى تجزيهى آمارى، امتيازدهى به به باندهاى واضح به به

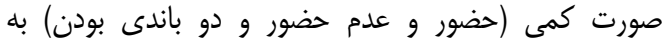

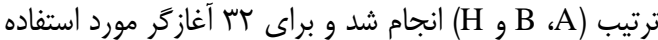
يك ماتريس تشكيل داده شد.

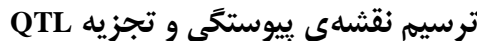

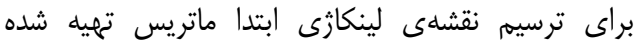

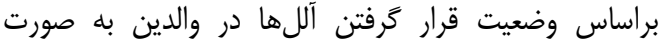

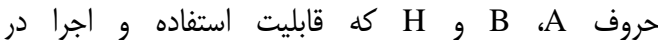

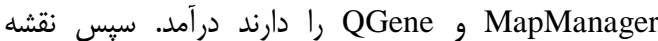
ترسيم شد. نقشهيابى فاصلهاى مركب (CIM) براى دادهاد

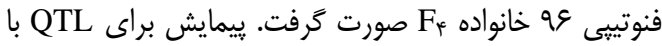

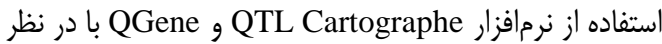

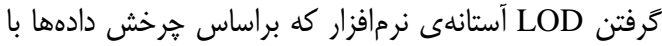

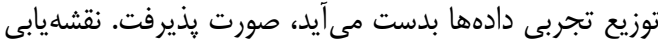

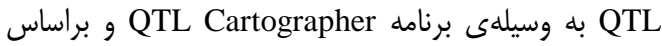

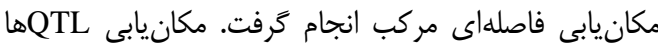

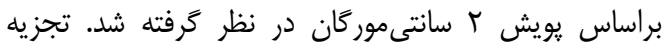

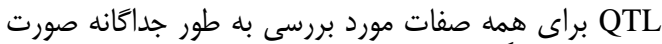

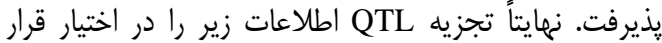

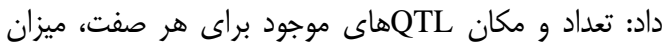

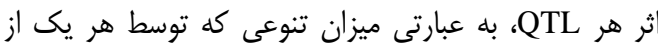

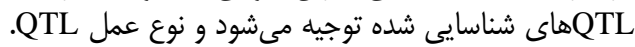

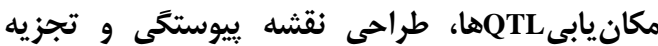
آمارى

Map نقشه لينكازى ريزماهوارهها با استفاده از نرمافزار

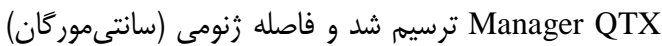

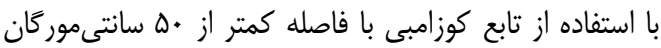

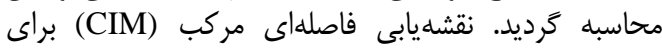

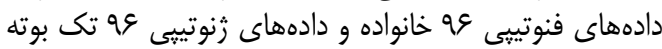

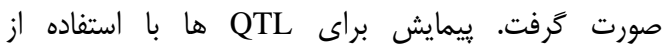

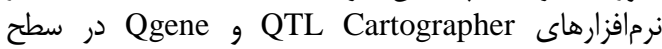
احتمال ال درصد انجام شد.
تاثير قرار مىدادند روى اين كروموزوم كزارش مران كردهاند.

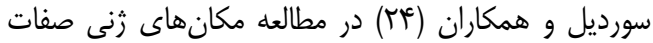

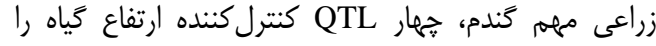

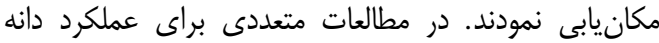

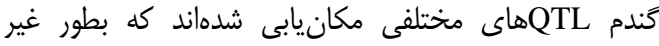

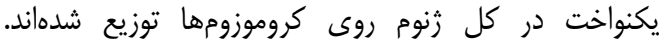

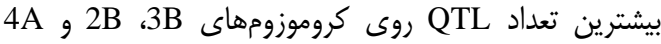

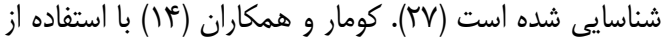

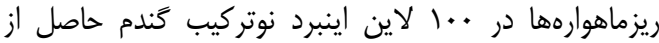
تلاقى ارقام PH132 و و

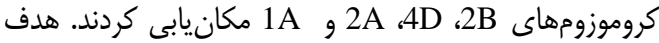

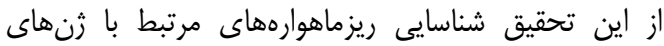

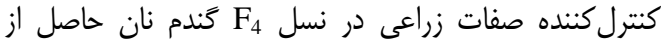

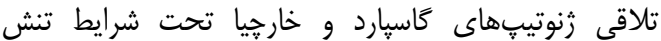

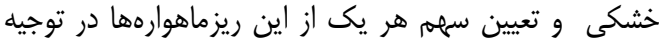

تنوع فنوتييى صفات مذكور بود.

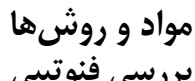

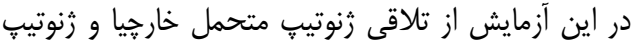

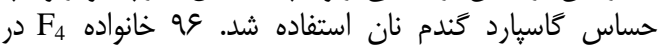

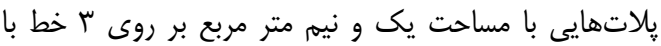

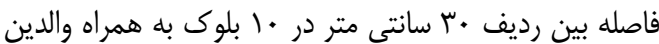

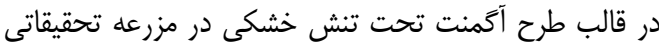

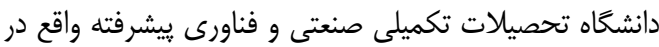

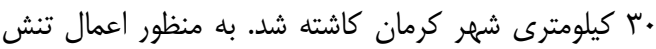

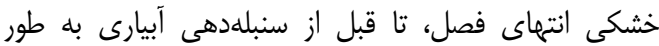

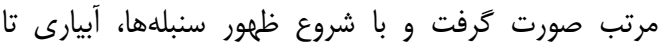

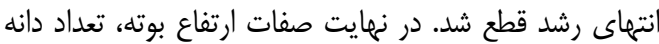

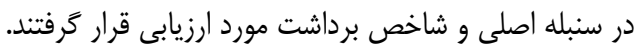
ارزيابى زنوتيبى

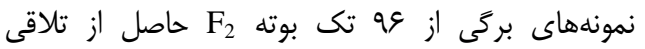

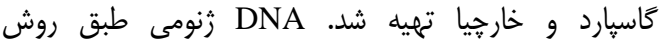

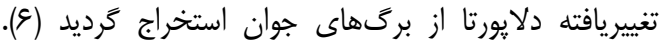

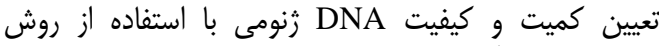

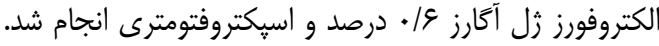

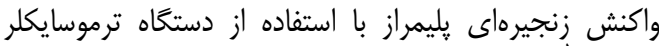

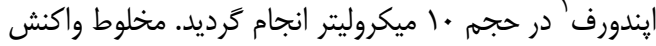

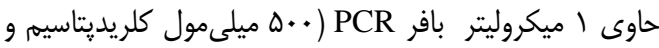

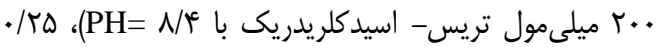

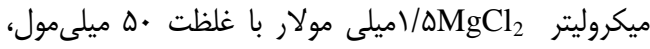

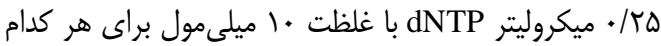

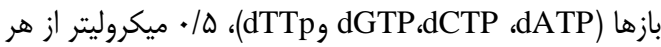

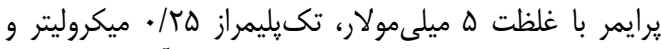

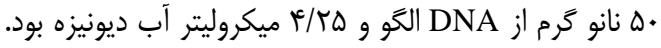

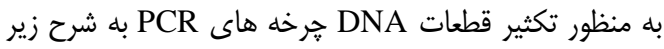

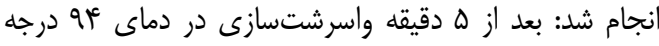

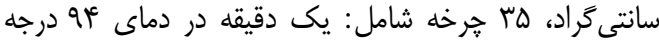




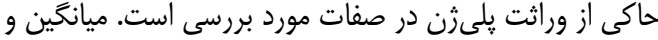

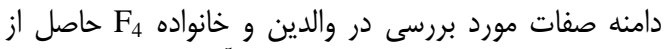

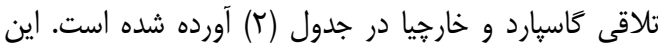

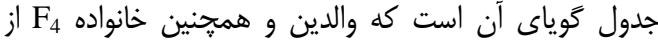
نظر صفات مورد مطالعه متفاوت بوده و تنوع دارند.
نتايج و بحث

نتايج ارزيابى فنوتيبى

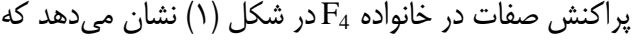

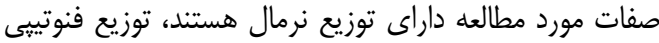

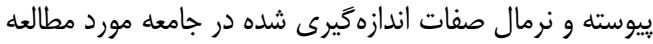

جدول ا- اسامى ريزماهوارهها، دماى اتصال، مكان كروموزومى و طول قطعه نشانكرهاى جندشكل در سطح والدين مورد استفاده در گندم نان Table 1. Microsatellite marker names, annealing temperature, chromosomal location and length of a piece of multimodal markers at the level of parents used in bread wheat

\begin{tabular}{|c|c|c|c|c|}
\hline نشانگر ريزماهواره & دماى اتصال & توالى تكرارى( موتيف) & مكان كروموزومى & طول قطعه \\
\hline Xgwm6 & 55 & $(\mathrm{GA}) 40$ & $4 \mathrm{~B}$ & 196-207bp \\
\hline Xgwm44 & 60 & (GA) 28 & 7D & 176-178bp \\
\hline Xgwm46 & 60 & (GA) $2 \mathrm{GC}(\mathrm{GA}) 33$ & $7 \mathrm{~B}$ & $176-179 b p$ \\
\hline Xgwm102 & 60 & (CT) 15 & $2 \mathrm{D}$ & $145-153 b p$ \\
\hline Xgwm114 & 60 & (GA) 53 & 3B & $142-168 b p$ \\
\hline Xgwm148 & 55 & (CA) 22 & 2B & $152-167 b p$ \\
\hline Xgwm186 & 60 & (GA)26 & $5 \mathrm{~A}$ & $106-132 b p$ \\
\hline Xgwm249 & 55 & (GA) $11(\mathrm{GGA}) 8$ & $2 \mathrm{D}$ & $150-154 b p$ \\
\hline Xgwm251 & 55 & (CA) 28 & 4B & 109-110bp \\
\hline Xgwm 257 & 60 & (GT) 30 & $2 \mathrm{~B}$ & 190-192bp \\
\hline Xgwm261 & 55 & (CT) 21 & $2 \mathrm{D}$ & 164-194bp \\
\hline Xgwm271 & 60 & (CT)4imp(GA) 10 & $5 \mathrm{D}$ & $179 b p$ \\
\hline Xgwm274 & 50 & (GT) 27 & $1 \mathrm{~B}, 7 \mathrm{~B}$ & $177-184 b p$ \\
\hline Xgwm285 & 60 & (GA) 27 & 3B & $222-227 b p$ \\
\hline Xgwm295 & 60 & (GA) 25 & $7 \mathrm{D}$ & $254-258 b p$ \\
\hline Xgwm297 & 55 & (GT) $12(\mathrm{GA}) 18$ & 7B & $150-168 b p$ \\
\hline Xgwm 320 & 55 & (GT) $9(\mathrm{GA}) 15$ & $2 \mathrm{D}$ & $226 \mathrm{bp}$ \\
\hline Xgwm332 & 60 & (GA) 36 & $7 \mathrm{~A}$ & $290-211 b p$ \\
\hline Xgwm340 & 60 & (GA)26 & 3B & $159 b p$ \\
\hline Xgwm 350 & 55 & (GT) 14 & $7 \mathrm{~A}$ & 209-215bp \\
\hline Xgwm 368 & 60 & (AT) 25 & 7B & $259-271 b p$ \\
\hline Xgwm 400 & 60 & (CA) 21 & $7 \mathrm{~B}$ & $143-150 b p$ \\
\hline Xgwm408 & 55 & $(\mathrm{CA})>22(\mathrm{TA})(\mathrm{CA}) 7(\mathrm{TA}) 9$ & $5 \mathrm{~B}$ & $148-182 b p$ \\
\hline Xgwm428 & 60 & $(\mathrm{GA}) 22$ & $7 \mathrm{D}$ & $133-137 b p$ \\
\hline Xgwm437 & 50 & (CT) 24 & $7 \mathrm{D}$ & $109-111 b p$ \\
\hline Xgwm484 & 55 & (CT) 29 & $2 \mathrm{D}$ & 43-153bp \\
\hline Xgwm495 & 60 & (GA) 20 & 4B & $160-178 b p$ \\
\hline Xgwm539 & 60 & (GA) 27 & $2 \mathrm{D}$ & 143-157bp \\
\hline Xgwm565 & 60 & (CA) 10 & $5 \mathrm{D}$ & 142-150bp \\
\hline Xgwm601 & 60 & (CT) 17 & $4 \mathrm{~A}$ & $142-152 b p$ \\
\hline Xgwm608 & 60 & (GA) 16 & $4 \mathrm{D}$ & 144-151bp \\
\hline Xgwm613 & 60 & (CT) 23 & $6 \mathrm{~B}$ & 114-118bp \\
\hline
\end{tabular}




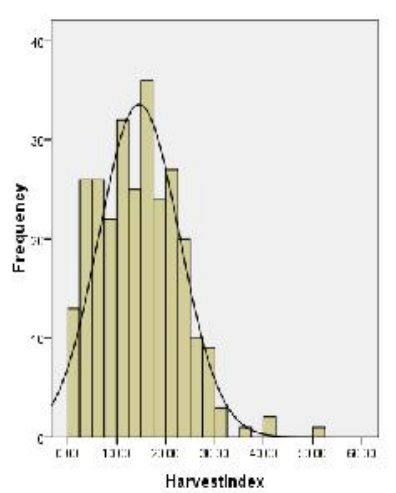

ج

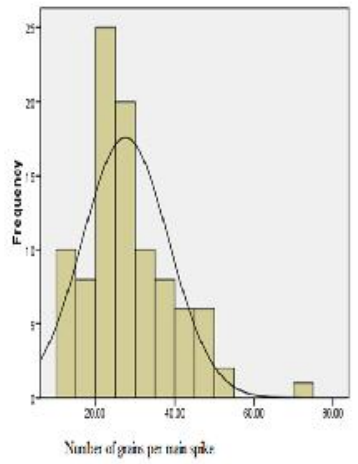

$\varphi$

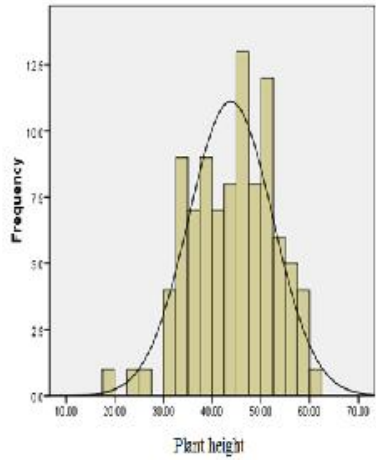

الف

شكل ا- توزيع فراوانى صفات زراعى در شرايط تنش خشكى در نسل F4: (الف) ارتفاع گياه، (ب) تعداد دانه در سنبله اصلى، (ج) شاخص برداشت

Figure 1. Frequency distribution of different traits in drought stress conditions in F4 generation a) Plant height, b) Grain weight per main spike, c) Harvest index

جدول r- مقايسه ميانگ̌ين صفات مختلف در شرايط تنش خشكى در والدين گَاسيارد، خارجيا و نسل Table 2. Comparison of the mean of different traits in drought stress conditions in parents of Gaspard, Charchia and

\begin{tabular}{|c|c|c|c|c|c|c|}
\hline \multirow[t]{2}{*}{ صفات كياهى } & \multicolumn{2}{|c|}{ P1 } & \multicolumn{2}{|c|}{ P2 } & \multicolumn{2}{|c|}{ F4 } \\
\hline & ميانكين & دامنه & ميانگين & دامنه & ميانغين & دامنه \\
\hline إرتفاع كياه & $F \mid / 9 Q$ & $r \Delta$ & $\mu F / \Lambda \Delta$ & $T N / \Delta$ & $r \psi / \Lambda 1$ & $f 1 / \Delta$ \\
\hline تعداد دانه در سنبله اصلى & $r y / q 9$ & $\Delta F$ & $I V / A V$ & ra & TV/DT & q. \\
\hline شاخص برداشت & $|\pi / r|$ & $\Delta F / . r$ & IT/T & $r q / / q$ & $10 / 4 y$ & $(q 9 / 4)$ \\
\hline
\end{tabular}

جدول س- - خاى شناسايى شده به روش مكانيابى فاصلهاى مركب براى صفات مختلف در جمعيتهاى نسل جهارم در شرايط تنش خشكى در كندم نان

Table 3. Identified QTLs by mapping composite interval method for different traits in fourth generation populations

\begin{tabular}{|c|c|c|c|c|c|c|c|}
\hline QTL & اثر غالبيت & اثر افزايشى & $\mathrm{R}^{2}$ & كروموزوم & LOD & موقعيت & صفت \\
\hline QH-7B-a & $-\{N / \backslash A \mid$ & -TYT/QGV & . & $\Gamma / Q \wedge \Delta$ & $7 \mathrm{~B}$ & $r \cdot$ & \multirow{3}{*}{ ارتفاع كَياه } \\
\hline QH-7B-b & KITF/TH & $-I V T / V N S$ & זוM & r/৭९9 & 7B & rr & \\
\hline QH-7B-c & 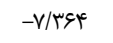 & $1 / \cdot r$ & سזא/ & $r / r I I$ & $7 \mathrm{~B}$ & fif & \\
\hline QNGPMS-2B-a & $-r r \cdot N / M$ & $-1119 / \mathrm{WV}$ &.$/ 1$ & $r / \Delta$ & $2 \mathrm{~B}$ & r. & تعداد دانه در سنبله اصلى \\
\hline QHI-4B-a & $-|F \Delta| K \mid$ & $-F \mid \Delta \Delta / \& \Lambda$ & . /NF & r/194 & $4 \mathrm{~B}$ & r. & شاخص برداشت \\
\hline
\end{tabular}




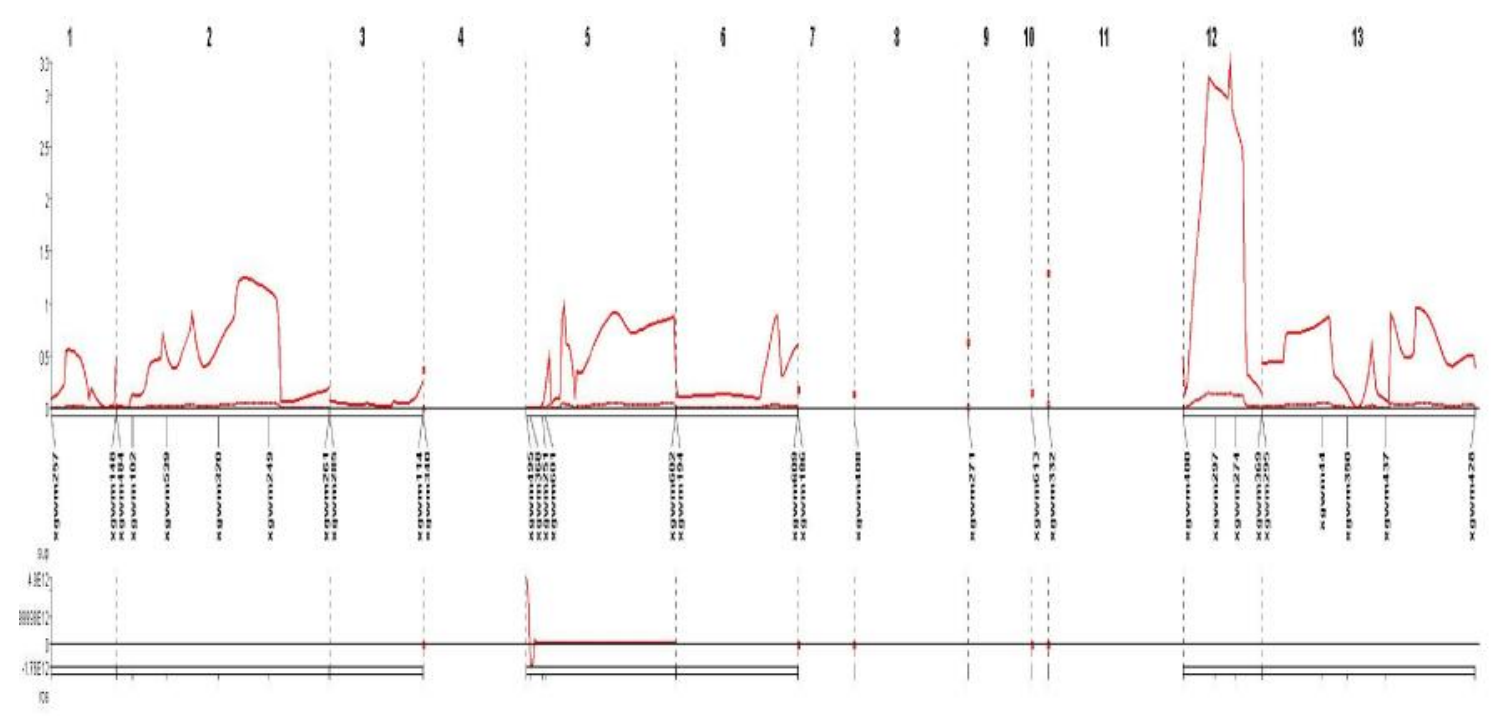

شكل rQ - هاى مكان يابى شده در برخى كروموزومها براى صفت ارتفاع بوته

Figure 2. QTLs Found on some chromosomes for Plant Height

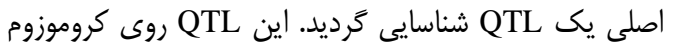

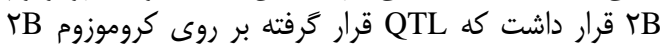

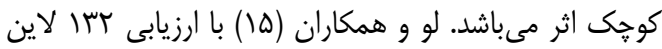

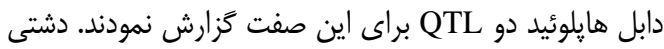

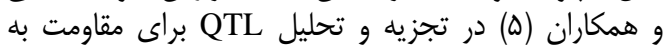

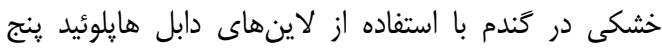

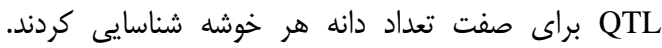

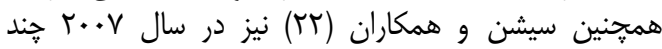

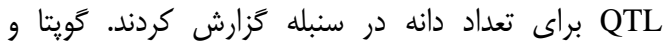

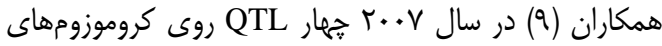

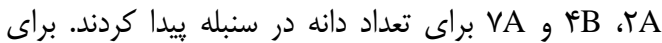

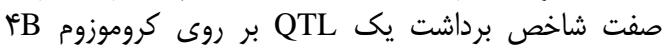

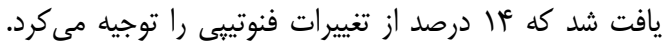

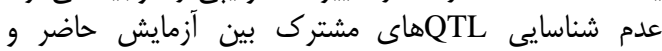

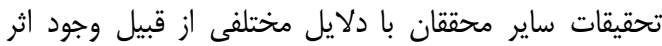

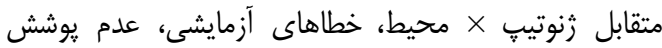

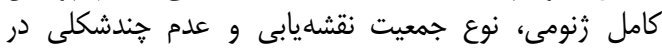

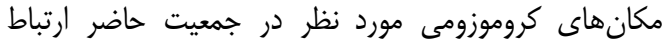

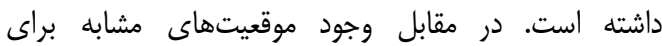

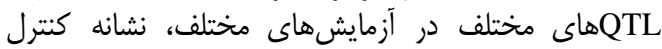

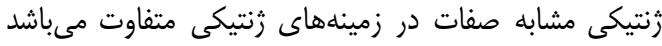

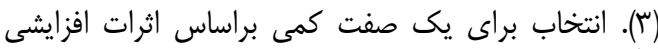

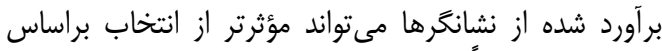

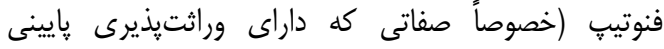

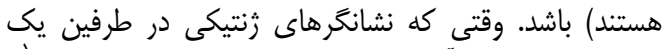

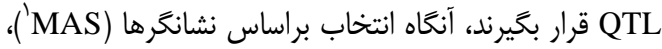

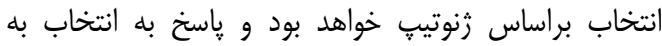

نتايج تجزيه QTL

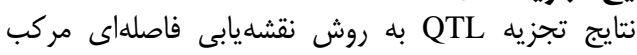

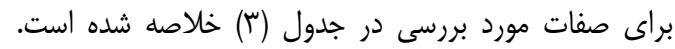

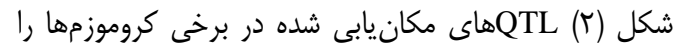

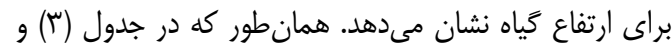

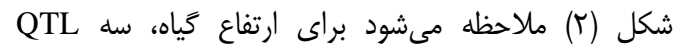

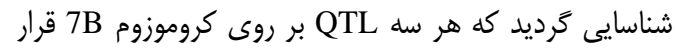

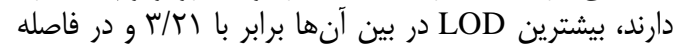

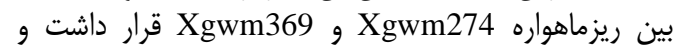

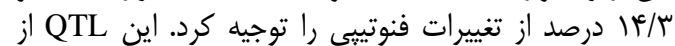

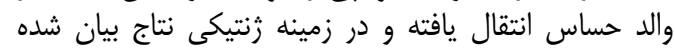

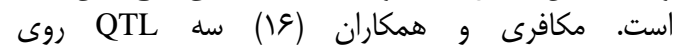

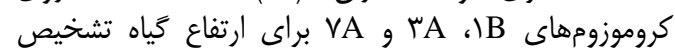

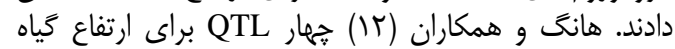

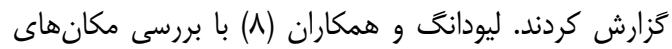

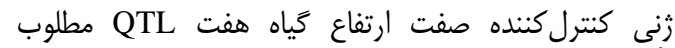

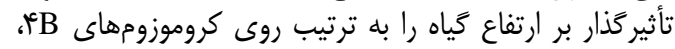

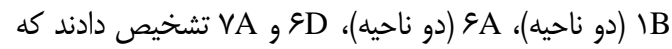

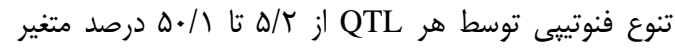

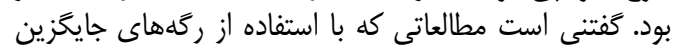

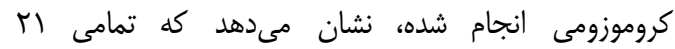

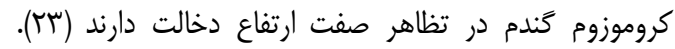

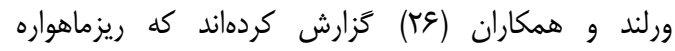

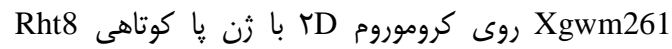

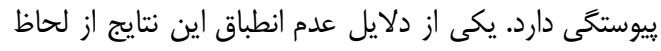

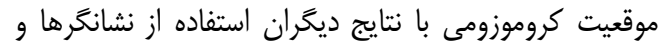
جمعيت مختلف مىباشد. براى صفت تعداد دان دانه در سنبله 


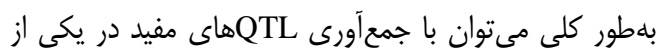

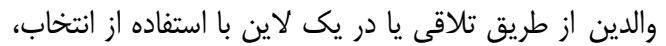

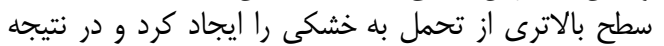

عملكرد بالاترى را درشرايط تنش خشكى بله به دست آرود.

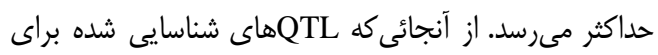

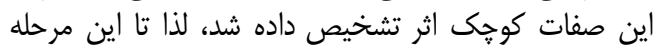

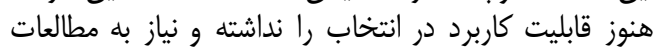

بيشتر در شرايط محيطى مىباشد.

1. Abdul, Q., R. Abdul, A. Muhammad and M.A. Jenks. 2011. Water stress causes differential effects on germination indices, total soluble sugar and proline content. African Journal of Biotechnology, 10: $14038-14045$.

2. Arif, M. 2002. Molecular mapping of genes/QTLs affecting resistance to Xanthomonas oryza $p v$. Oryza and grain quality traits in rice (Oryza sativa L.). PhD thesis, University of Philippines in Los Banos, Philippines.

3. Blanco, A., R. Simeone and A. Gadaleta. 2006. Detection of QTLs for grian protein content in durum wheat. Theoretical and Applied Genetics, 112: 1195-1204.

4. Bryan, G.J., A.J. Collins, P. Stephenson, A. Orry and J.B. Smith. 1997. Isolation and characterization of microsatellite from hexaploid bread Wheat. Theoretical and Applied Genetics, 94: 557-563.

5. Dashti, H., B. Yazdi Samadi, S. Abdmyshany and M.R. Ghannadha. 2001. QTL analysis of cold tolerance traits and heading date in wheat using double Haploids Journal of Agricultural Science, 32: 177-157 (In Persian).

6. Dellaporta, S.L., J. Wood and J.B. Hicks. 1983. A plant DNA minipreparation: Version II. Plant Molecular Biology Reporter, 4: 19-21.

7. Diab, A.A., A.H. Fahmy, O.S. Hassan, M.M. Nachit and O.A. Momtaz. 2007. Identification of chromosomal region and genetic contribution of gene controlling yield and other agronomic traits in durum wheat grown under different Egyptian environmental conditions. World Journal of Agricultural Sciences, 3: 401-422.

8. Dong-Cheng, L., G. Mu-Qiang, G. Rong-Xia, L. Run-Zhi, C. Shuang-He, L. Xiao-Li and A.M. Zhang. 2002. Mapping Quantitative Trait Loci for Plant Height in Wheat (Triticum aestivum L.) Using a $F_{2: 3}$ Populations, Journal of Genetics and Genomics, 29(8): 706-71.

9. Gupta, P.K., H.S. Balyan, P.L. Kulwal, N. Kumar, A. Kumar, R.R. Mir, A. Mohan, J. Kumar and J. Zhejiang. 2007. QTL analysis for some quantitative traits in bread wheat. University Science B, 8: 807-814.

10. Gupta, P.K., H.S. Balyan, K.J. Edwards, P. Isaac, V. Korzun, M.S. Roder, F. Gautier, P. Joudrier, A.R. Schlatter, J. Dobcovsky, R. Delapena, M. Khairallah, G. Penner, M.J. Hayden, P. Sharp, B. Keller, R.C. Wang, J.P. Hardouin, P. Jack and P. leory. 2002. Genetic mapping of 66 new microsatellite (SSR) loci in bread wheat, Theoretical and Applied Genetics, 105: 413-422.

11. Hassan, S.E. and I. Khaliq. 2008. Quantitative inheritance of some physiological traits for spring wheat under two different population densities. Pakistan Journal of Botany, 40: 581-587.

12. Huang, X.Q., S. Cloutier, L. Lycar, N. Radovanovic, D.G.N.J.S. Humphreys, D.J. Somers and P.D. Brown. 2006. Molecular detection of QTLs for agronomic and quality traits in a doubled haploid population derived from two Canadian wheats (Triticum aestivum L.), Theoretical and Applied Genetics, 113: 753-766.

13. Kirigwi, F.M., M. Van Ginkel, R.G. Trethowan, R.G. Sears, S. Rajaram and G.M. Paulsen. 2004. Evaluation of selection strategies for wheat adaptation across water regimes. Euphytica, 135: 361-367.

14. Kumar, A. and S.C. Sharma. 2007. Genetics of excised-leaf water loss and relative water content in bread wheat (Triticum aestivum L.). Cereal Research Communications, 1: 43-52.

15. Lu, C., L. Shen, Z. Tan, Y. Xu, Y. Chen and L. Zhu. 1997. Comparative mapping of QTLs for agronomic traits of rice across environments using a doubled-haploid population, Theoretical and Applied Genetics, 93: 1211-1217.

16. Maccaferri, M., M.C. Sanguineti, S. Corneti, J.L.A. Ortega, M.B. Salem, J. Bort, E. Deambrogio, L.F.G. Moral, A. Demontis, A. Eh-Ahmed, F. Maalouf, H. Machlab, V. Martos, M. Moragues, J. Motawaj, M. Nachit, N. Nserallah, H. Ouabbou, C. Royo, A. Slama. 2008. Tuberosa, Quantitative trait loci for grain yield and adaptation of durum wheat (Triticum durum Desf.) across a wide range of water_availability, Genetics, 178: 489-511.

17. MacKill, D.J. 2003. What molecular tools are available for selection for drought tolerance? In: Fischer, K. S., Laffite, R., and Fukai, S. (eds.) Environments. IRRI, Philippines, 55-61.

18. Price, A. and B. Courtois. 1999. Mapping QTLs associated with drought resistance in rice: progress, problems and prospects. Plant Growth Regulators, 29: 123-133.

19. Roder, M.S., V. Korzun, K. Wendehake, J. Plaocke, M.H. Tixier, P. Lerou and M.W. Ganal. 1998. A microsatellite map of wheat. Genetics, 144: 2007-2023.

20. Sami, U.A., A.S. Khan, A. Raza and S. Sadique. 2010. Gene action analysis of yield and yield related traits in spring wheat (Triticum aestivum). International Journal of Agriculture Biology, 12: 125-128. 
21. Shah, M.M., K.S. Gill, P.S. Baenziger, Y. Yen, S.M. Kaeppler and H.H. Ariyarathne. 1999. Molecular mapping of loci for agronomic traits on chromosome 3A of bread wheat. Crop Science, 39: 17281732.

22. Sishen, L., J. Jizeng and W. Xyanyun. 2007. An interval genetic map and QTL analysis for height in wheat using a doubled-haploid population, Theoretical and Applied Genetics, 96: 933-940.

23. Snape, J.W., C.N. Law and A.J. Worland. 1977. Whole-chromosome analysis of height in wheat. Heredity $38: 25-36$.

24. Sourdille, P., T. Cadalen, H. Guyomarc, H.J.W. Snape, M.R. Perretant, G. Charmet, C. Boeuf, S. Bernard and M. Bernard. 2003. An update of the Courtot $\times$ Chinse spring intervarietal molecular marker linkage map for QTL detection of agronomic traits in wheat. Theoretical and Applied Genetics, 106: 530-538.

25. Trethowan, R.M., M. VanGinkel and S. Rajaram. 2002. Progress in breeding wheat for yield and adaptation in global drought affected environments. Crop Science, 42: 1441-1446.

26. Worland, A.J. and C.N. Law. 1986. Genetic analysis of chromosome 2D of wheat. Z Pflanzenzuencht, 96: 331-345.

27. Zhang, L.Y., D.C. Liu, X.L. Guo, W.L. Yang, J.Z. Sun, D. Wang and A. Zhang. 2009. Distribution in genome of quantitative trait loci (QTL) for yield and yield-related traits in common wheat (Triticum aestivum L.). Theoretical and Applied Genetics, 119: 43-52. 


\title{
Mapping of Loci Controlling Some of Agronomic Traits in Bread Wheat Under Drought Stress Conditions using Microsatellite Markers
}

\section{Jamileh Abedi $^{1}$, Ghasem Mohammadi Nejad ${ }^{2}$ and Amin Baghizadeh ${ }^{3}$}

1- Graduate Student, University of Advanced Technology, Kerman, Iran

2- Department of Agronomy and Plant Breeding, College of Agriculture, Shahid Bahonar University of Kerman, Kerman, Iran, (Corresponding author: Mohammadinejad@uk.ac.ir)

3- Department of Biotechnology, Institute of Science and High Technology and Environmental Sciences, Graduate University of Advanced Technology, Kerman-Iran Received: May 12, $2015 \quad$ Accepted: September 30, 2018

\begin{abstract}
In order to identify microsatellite markers linked to some traits of wheat under drought stress at the end of the season, the population included 96 family F2:4 genotypes derived from the cross Kharchia (tolerant parent) and Gaspard (susceptible parent) were evaluated for 2 years. Of the 92 microsatellite markers used to assess parents, 32 markers were polymorphic that located on chromosomes 2B, 2D, 3B, 4A, 4B, 4D, 5A, 5B, 5D, 6B, 7A, 7B, 7D. Based on composite interval mapping was found 3 QTL for plant height trait which were located on chromosome 7B and the highest QTL with the largest LOD equal to 3.21 was located between Xgwm274 and Xgwm369 and justified 14.3 percent of phenotypic variation. For number of seeds per main spike trait, 1 QTL was detected on chromosome 2B and 1 QTL was identified on the chromosome 4B for harvest index trait. The common position of some of the located QTLs indicates a genetic linkage or pleiotropic effect. The saturation of the desired map and the evaluation of the stability of the studied traits can confirm the ability to identify the controller QTLs and provide the basis for Marker-Assisted Selection.
\end{abstract}

Keywords: Bread Wheat, Drought Stress, QTL, SSR 